${ }^{1}$ Centro Cardiovascular, Hospital Clínico Regional de Concepción: "Dr. Guillermo Grant Benavente", Concepción, Chile. 2Departamento de Cirugía, Facultad de Medicina, Universidad de Concepción, Concepción, Chile. ${ }^{3}$ Facultad de Medicina, Departamento de Cirugía, Universidad de Concepción, Concepción, Chile. ${ }^{4}$ Unidad Neuromuscular, Servicio Neurología, Hospital Clínico Regional de Concepción: "Dr. Guillermo Grant Benavente", Concepción, Chile.

${ }^{5}$ Departamento de Especialidades, Facultad de Medicina, Universidad de Concepción, Concepción, Chile.

${ }^{6}$ Servicio de Cirugía, Hospital Clínico Regional de Concepción: "Dr. Guillermo Grant Benavente", Concepción, Chile. Interno Medicina, Facultad Medicina, Universidad de Concepción, Concepción, Chile.

No existen conflictos de interés ni apoyo financiero.

Recibido el 28 de septiembre de 2017, aceptado el 11 de abril de 2018.

Correspondencia a: Roberto González Lagos Departamento de Cirugía, Facultad de Medicina, Universidad de Concepción, Janequeo esquina Chacabuco S/N, Concepción, Chile. rgonzalezlagos@udec.cl

Material suplementario en versión digital de la Revista Médica de Chile en: https://scielo.conicyt.cl

\section{Miastenia gravis: resultados inmediatos y alejados de la timectomía transesternal extendida}

\author{
ROBERTO GONZÁLEZ L., ${ }^{1,2}$, ALEJANDRA RIQUELME U. ${ }^{3}$, \\ MARIO FUENTEALBA S. ${ }^{4,5}$, JORGE CANALES Z. ${ }^{\mathrm{a}}$, ALBERTO FUENTES E. ${ }^{a}$, \\ RENÉ SALDÍAS F. ${ }^{6}$, ENRIQUE SEGUEL S. ${ }^{1,2}$, ALECK STOCKINS L. ${ }^{1,2}$, \\ ANDRÉS JADUE T. ${ }^{1}$, EMILIO ALARCÓN C. ${ }^{1,2}$
}

\section{Results of extended transsternal thymectomy for Myasthenia gravis. Experience with 58 patients}

Background: Thymectomy improves clinical outcomes and decreases the need for medical treatment in patients with myasthenia gravis (MG). Aim: To describe the immediate and long-term results of extended transsternal thymectomy (ETT) in patients with MG. Material and Methods: A review of databases, surgical protocols, clinical records and interviews of patients subjected to extended transsternal thymectomy for MG between 1990 and 2016. Perioperative clinical characteristics, anticholinesterase treatment, immediate and remote surgical results were analyzed and patients were followed from one to 10 years. Results: We studied 58 patients aged $35 \pm 14$ years (72\%) women. In the preoperative period, according to Osserman classification, nine patients $(15,5 \%)$ were in grade I, eight (13,8\%) in grade IIA 8 and $40(69 \%)$ in grade IIB. The pathological study of the surgical piece showed thymic hyperplasia in 39 cases $(67,2 \%)$. Four patients had postoperative complications but none died. In the Follow-up at 1, 3, 5, 8 and 10 years the Masaoka palliation rate was 71.7, 77.5, 67.7, 70.0 and 70,6\% respectively. The figures for remission rate were 13.0, 15.0,19.4, 35.0 and 35,3\% respectively. The figures for Zielinski positive results were 79.6, 87.5, 87.1, 90.0 and 82,4\% respectively. The DeFilippi score improved by 80.4, 87.5, 87.1, 90.0 and 82.4\% respectively. The Myasthenia Gravis Foundation of America Post-Intervention State improved by 67.4, 77.5, 77.5, 75.0 and 70,6\% respectively. Mean Myasthenia Gravis Activities of daily living (MGADL) and Myasthenia Gravis Quality of life scale 15 (MGQOL 15) were 1.65 and 6.31 respectively. Conclusions: In selected patients with $M G$, extended transsternal thymectomy in MG has good immediate and long-term results.

(Rev Med Chile 2018; 146: 460-469)

Key words: Myasthenia Gravis; Thoracic Surgery; Thymectomy; Thymus Hyperplasia.

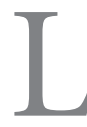
a miastenia gravis (MG) es una enfermedad autoinmune, que se caracteriza por la producción anormal de autoanticuerpos que afectan a la unión neuromuscular o placa motora, destruyendo los receptores de acetilcolina, lo que se manifiesta clínicamente como una progresiva debilidad en la musculatura estriada ${ }^{1}$.

La prevalencia de la MG se estima que es de 0,5 a 25 casos por 100.000 habitantes con una incidencia de 0,3 a 3 casos por 100.000 habitantes; 
suele afectar a individuos de todas las edades, sin embargo, existe una predilección por mujeres entre los 20 y 40 años; y en hombres entre 50 y 60 años. Clínicamente se caracteriza por tener una evolución progresiva o en brotes separados con períodos de remisiones de duración variable $e^{2-5}$.

La patogenia de esta enfermedad se basa en la producción de anticuerpos: acetylcholine receptor-antibodies (AChR), lipoprotein receptor-related protein 4 (LRP4) y muscle-specific tyrosine kinase (MuSK) que disminuyen considerablemente el número de receptores de acetilcolina en la unión neuromuscular, mediante 3 mecanismos: bloqueo del sitio activo de los receptores de acetilcolina, acelerada endocitosis de los receptores de acetilcolina y daño de la membrana post-sináptica mediado por complemento ${ }^{6,7}$.

En 1901 gracias a Oppenheim y Laquer-Weigert $^{8}$ surgen las primeras relaciones entre la MG y el timo, hoy en día está demostrado que el timo cumple un papel clave en la inducción y producción de anticuerpos ${ }^{9}$, además de su vinculación con alteraciones histológicas entre ellas las neoplasias (timomas) que pueden encontrarse entre $10 \%$ y $20 \%$ de los pacientes con MG, siendo en estos casos de peor pronóstico dado la mayor gravedad en los síntomas y menor respuesta a tratamiento ${ }^{10}$.

En 1911, Sauerbruch realizó la primera timectomía en un paciente con MG, con el cual obtuvo una gran mejoría de los síntomas, pero no es hasta 1936 cuando Blalock publicó una serie de 6 casos en los cuales se les realizó timectomía obteniendo resultados favorables ${ }^{11-13}$, desde entonces se han publicado múltiples series exponiendo los beneficios de la extirpación del timo en la evolución de la MG, dando lugar a múltiples controversias entre ellas: indicaciones, abordajes quirúrgicos y momento en que se debe efectuar la timectomía.

Se han desarrollado múltiples técnicas quirúrgicas con el fin de remover el timo: timectomia transesternal extendida (TTEE), cervicotomía, timectomia por toracoscopía video asistida, entre otras. La TTEE es la que mejores resultados ha tenido gracias a la exposición que da, lo que permite maximizar la exéresis de tejido tímico con una baja morbilidad $^{14-17}$.

Algunos autores han cuestionado los beneficios de la timectomía por sobre la terapia médica debido a que la mayoría de las series son retrospectivos-observacionales con una falla en la metodología (principalmente en las definiciones de remisión), que al compararlos no permitían conclusiones definitivas $^{18,19}$. Sin embargo, en agosto de 2016 Wolfe et al. ${ }^{20}$, publicó los resultados largamente esperados del primer ensayo clínico internacional, aleatorizado y controlado de la timectomía en MG (MGTX), en donde se compararon los beneficios de la TTEE versus el tratamiento médico en pacientes con MG no timomatosa, demostrando la timectomía mejores resultados tanto en los objetivos primarios (mejoría clínica y dosis requerida de prednisona) como en la mayoría de los resultados secundarios (uso de azatioprina, tasa de hospitalización y proporción de pacientes con mínimas manifestaciones). Además, se comunicó recientemente que se produjo un error al calcular el promedio de la dosis de prednisona ponderada en el tiempo en los pacientes que se suspendió su uso; a pesar de lo anterior el cambio de los resultados primarios, secundarios y las conclusiones del ensayo no se alteran de la versión original publicada, sino por el contrario, refuerzan el valor terapéutico de la timectomía ${ }^{21}$.

Los objetivos del presente trabajo son caracterizar los pacientes y presentar los resultados inmediatos y alejados de la TTEE realizada a los pacientes con MG.

\section{Material y Método}

Se realizó un estudio descriptivo longitudinal de los pacientes timectomizados con diagnóstico de MG en el Hospital Clínico Regional "Dr. Guillermo Grant Benavente" de Concepción-Chile operados por el Equipo de Cirugía Cardiotorácica, en el período comprendido entre enero de 1990 y diciembre de 2016.

Los datos recolectados se obtuvieron a partir protocolos de MG realizados al momento de ingreso al hospital, revisión de base de datos, protocolos quirúrgicos, fichas clínicas, registro civil, entrevistas clínicas y telefónicas. Esta comunicación se presentó al Comité de Ética de la Facultad de Medicina de la Universidad de Concepción.

El abordaje quirúrgico en nuestro centro fue el transesternal extendido, siguiendo las recomendaciones internacionales ${ }^{14,15}$.

Se describe y compara características clínicas 
perioperatorias, tratamiento anticolinesterásico, resultados quirúrgicos inmediatos y alejados. Se realizó un seguimiento hasta 10 años utilizando las siguientes clasificaciones: Masaoka ${ }^{15}$, Zielins$\mathrm{ki}^{22}$, DeFilippi ${ }^{23}$ y Myasthenia Gravis Foundation of America Post intervention Status (MGFA-PIS) ${ }^{14}$, registrándose los resultados a 1, 3, 5, 8 y 10 años post timectomía; además se aplicaron las siguientes encuestas en el último control y/o entrevista: Myasthenia Gravis Activities of Daily Living $(M G A D L)^{24}$ y Myasthenia Gravis Quality of Life scale 15 (MGQOL 15) ${ }^{25}$ (Anexo 1).

El procesamiento de información fue efectuado en Microsoft Excel ${ }^{\circledR} 2016$ mediante el uso de planillas de recolección de datos para posterior análisis, utilizando medidas de tendencia central y análisis porcentual, además, se realizó estudio de regresión cuadrática para análisis de dosificación de anticolinérgico pre y post TTEE, calculando $\mathrm{R}^{2}$, se consideró significativo $\mathrm{p}<0,05$.

\section{Resultados}

En el período, 58 pacientes con MG fueron timectomizados. Dentro de las características epidemiológicas del grupo destaca un predomino de la población femenina (relación F/M: 2,6/1), adulto joven (promedio de edad de 35,4 años). En cuanto a las patologías asociadas, $22(37,9 \%)$ de ellos presentaban alguna comorbilidad siendo las principales: hipertensión arterial, tabaquismo y obesidad, además se observó que 5,2\% de los pacientes tenían otras enfermedades autoinmunes (Tabla 1).

El tiempo promedio de evolución de la MG hasta la timectomia fue de 32,4 meses, destacando que la mitad de ellos fueron operados antes del año desde el diagnóstico de la MG; además se registraron 17 pacientes con crisis miasténicas previo a la cirugía. Al utilizar la clasificación de Osserman el subtipo IIB fue el más frecuente, seguido del tipo I (Tabla 2).

Las manifestaciones clínicas más frecuentes que presentaban los pacientes previos a la cirugía fueron ptosis palpebral, diplopía y disfagia (Tabla $3)$. El tratamiento médico preoperatorio fue principalmente monoterapia con anticolinesterásicos o bien asociado al uso de prednisona o algún otro inmunosupresor como la azatioprina, solo una paciente se encontraba sin medicación dado la intensidad de los efectos adversos de estos (Tabla 3 ).
Tabla 1. Número, edad, sexo y comorbilidades en pacientes timectomizados por miastenia gravis

\begin{tabular}{|c|c|}
\hline \multicolumn{2}{|l|}{ Características } \\
\hline Total de pacientes & 58 \\
\hline \multicolumn{2}{|l|}{ Edad (años) } \\
\hline Promedio & $35,4 \pm 13,9$ \\
\hline Mediana & 34,5 \\
\hline Rango & $14-74$ \\
\hline \multicolumn{2}{|l|}{ Sexo } \\
\hline Femenino & $42(72,4 \%)$ \\
\hline Masculino & $16(27,6 \%)$ \\
\hline Comorbilidades & $22(37,9 \%)$ \\
\hline Hipertensión arterial & $7(12,1 \%)$ \\
\hline Tabaquismo & $6(10,4 \%)$ \\
\hline Obesidad & $4 \quad(6,9 \%)$ \\
\hline Asma & $3(5,2 \%)$ \\
\hline Enfermedad autoinmune & $3(5,2 \%)$ \\
\hline Síndrome de Sjögren & $1 \quad(1,7 \%)$ \\
\hline Hipotiroidismo & $1 \quad(1,7 \%)$ \\
\hline Anemia perniciosa & $1 \quad(1,7 \%)$ \\
\hline Diabetes mellitus 2 & $2(3,4 \%)$ \\
\hline Enfermedad renal crónica en hemodiálisis & $2(3,4 \%)$ \\
\hline
\end{tabular}

Tabla 2. Características de la miastenia gravis en pacientes timectomizados

\begin{tabular}{|lc|}
\hline Total miastenia gravis & 58 \\
\hline Tiempo de evolución en Miastenia gravis (meses) \\
Promedio & $32,4 \pm 48,9$ \\
No timoma & $37,9 \pm 52,1$ \\
Timoma & $6,2 \pm 7,1$ \\
Mediana & 12 \\
Rango & $0,2-240$ \\
$\leq 12$ meses & $29(50 \%)$ \\
$>12-24$ meses & $13(13 \%)$ \\
$>24-60$ meses & $7(12,1 \%)$ \\
$>60$ meses & $9(15,5 \%)$ \\
Clasificación Osserman & \\
I & $9(15,5 \%)$ \\
IIA & $8(13,8 \%)$ \\
IIB & $40(69,0 \%)$ \\
III & $1(1,7 \%)$ \\
IV & 0 \\
Crisis de MG preoperatoria & $17(28,3 \%)$ \\
1 Crisis & $11(19,0 \%)$ \\
2 Crisis & $5(8,6 \%)$ \\
5 Crisis & $1(1,7 \%)$ \\
\hline
\end{tabular}


Tabla 3. Síntomas, signos y tratamiento farmacológico previos a la tímectomía en pacientes con Miastenia gravis

\begin{tabular}{|lc|}
\hline & $\mathbf{n}$ \\
\hline Síntomas y signos & \\
Ptosis palpebral & $39(67,2 \%)$ \\
Diplopía & $28(48,3 \%)$ \\
Disfagia & $23(39,7 \%)$ \\
Debilidad muscular generalizada & $19(32,8 \%)$ \\
Debilidad extremidades superiores & $16(27,6 \%)$ \\
Disartria & $14(24,1 \%)$ \\
Disnea & $12(20,7 \%)$ \\
Parálisis Facial & $9(15,5 \%)$ \\
Trastornos de la fonación & $3(5,2 \%)$ \\
Dolor torácico & $3(5,2 \%)$ \\
Otros & $4(6,9 \%)$ \\
\hline Fármacos & \\
Anticolinesterásicos & $39(67,2 \%)$ \\
Anticolinesterásicos + Corticoides & $17(29,3 \%)$ \\
10 mg diarios de prednisona & $6(10,3 \%)$ \\
$20-40$ mg diarios de prednisona & $10(17,2 \%)$ \\
Anticolinesterásicos + Inmunosupresión & $1(1,7 \%)$ \\
\hline
\end{tabular}

A la totalidad de los pacientes de nuestra serie se les realizó una TTEE, siendo los hallazgos histológicos los descritos en la Tabla 4. En relación a los timomas todos ellos se operaron con intención resectiva y solo uno quedó R1 (márgenes de pieza quirúrgica con células tumorales a la microscopia) y el resto R0 (resección tumoral macro y microscópica completa).

Los resultados inmediatos post quirúrgicos se visualizan en la Tabla 4 , destacando que $4(6,9 \%)$ pacientes presentaron alguna morbilidad post operatoria, de los cuales solo $1(1,7 \%)$ requirió ser reoperado a causa de un sangrado post operatorio y no necesitó transfusiones. No se registró mortalidad.

En relación a los resultados alejados post timectomía se logró un seguimiento de nuestros pacientes en $81 \%, 76 \%, 72 \%, 59 \%$ y $57 \%$ a 1,3 , 5,8 y 10 años de post operados respectivamente.
Tabla 4. Hallazgos histopatológicos y resultados inmediatos de la timectomía transesternal extendida

\begin{tabular}{|lc|}
\hline & $\mathbf{n}$ \\
\hline Histología & \\
Hiperplasia tímica & $39(67,2 \%)$ \\
Timoma & $10(17,2 \%)$ \\
Atrofia tímica & $9(15,5 \%)$ \\
\hline Peso* & \\
Promedio & $70,8 \pm 37,3 \mathrm{~g}$ \\
Morbilidad post operatoria & $4(6,9 \%)$ \\
Hemorragia post operatoria & $1(1,7 \%)$ \\
Crisis miasténica & $1(1,7 \%)$ \\
Infección de herida post operatoria & $1(1,7 \%)$ \\
Neumonía & $1(1,7 \%)$ \\
Reoperaciones & $1(1,7 \%)$ \\
Necesidad de transfusiones & 0 \\
Mortalidad & 0 \\
\hline Estadía post operatoria (días) & \\
Promedio & 10,2 \\
Mediana & 7 \\
\hline Estadía en UCl (días) & 3,1 \\
Promedio & 1 \\
Mediana & \\
\hline
\end{tabular}

*Tejido resecado, excluidos los timomas.

Se registraron $6(10,3 \%)$ pacientes fallecidos durante el seguimiento, de los cuales $1(1,7 \%)$ murió a causa de una crisis miasténica y $1(1,7 \%)$ de timoma, mientras que los 4 restantes fallecieron de otras causas tales como sepsis, cáncer vesicular entre otros.

Se observó una disminución significativa de la dosis promedio de anticolinesterásico entre la dosis preoperatoria y el seguimiento (Figura 1).

Los resultados clínicos alejados se muestran de acuerdo a las diferentes clasificaciones en las Figuras 2-4, destacando que se observa una mejoría importante independiente de la clasificación utilizada.

Al aplicar las encuestas de Myasthenia Gravis Activities of Daily Living (MGADL) y Myasthenia Gravis Quality of Life scale 15 (MGQOL 15) arrojaron puntajes promedio 1,65 y 6,31 puntos respectivamente (Figura 5). 

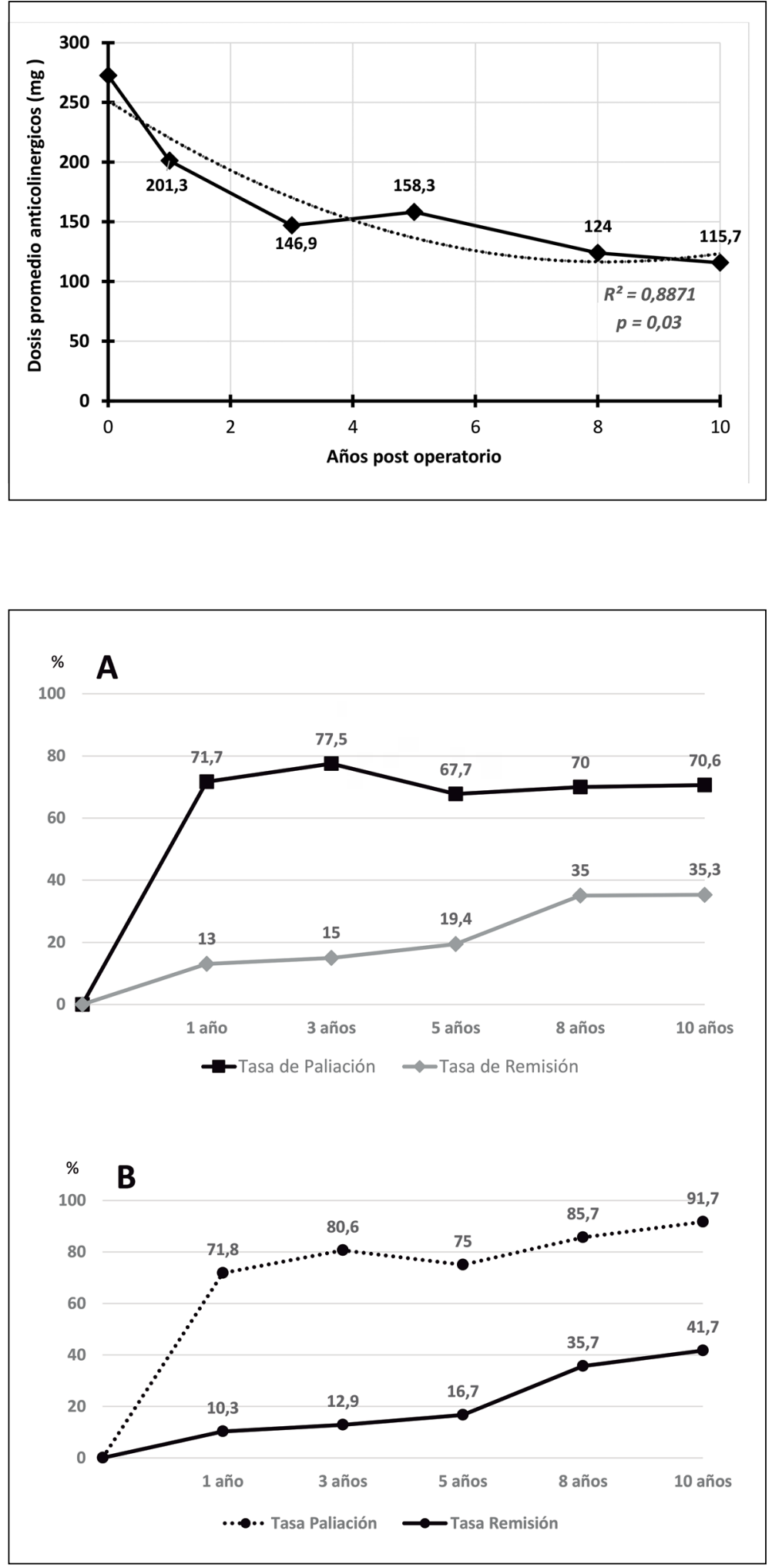

Figura 1. Evolución de la dosis promedio de anticolinesterásico post timectomía.
Figura 2. A. Tasa de paliación y remisión de pacientes timectomizados, según clasificación de Masaoka ${ }^{15}$ a 10 años post timectomía. B. Tasa de paliación y remisión de pacientes timectomizados no timomatosos, según clasificación de Masaoka ${ }^{15}$ a 10 años post timectomía. 

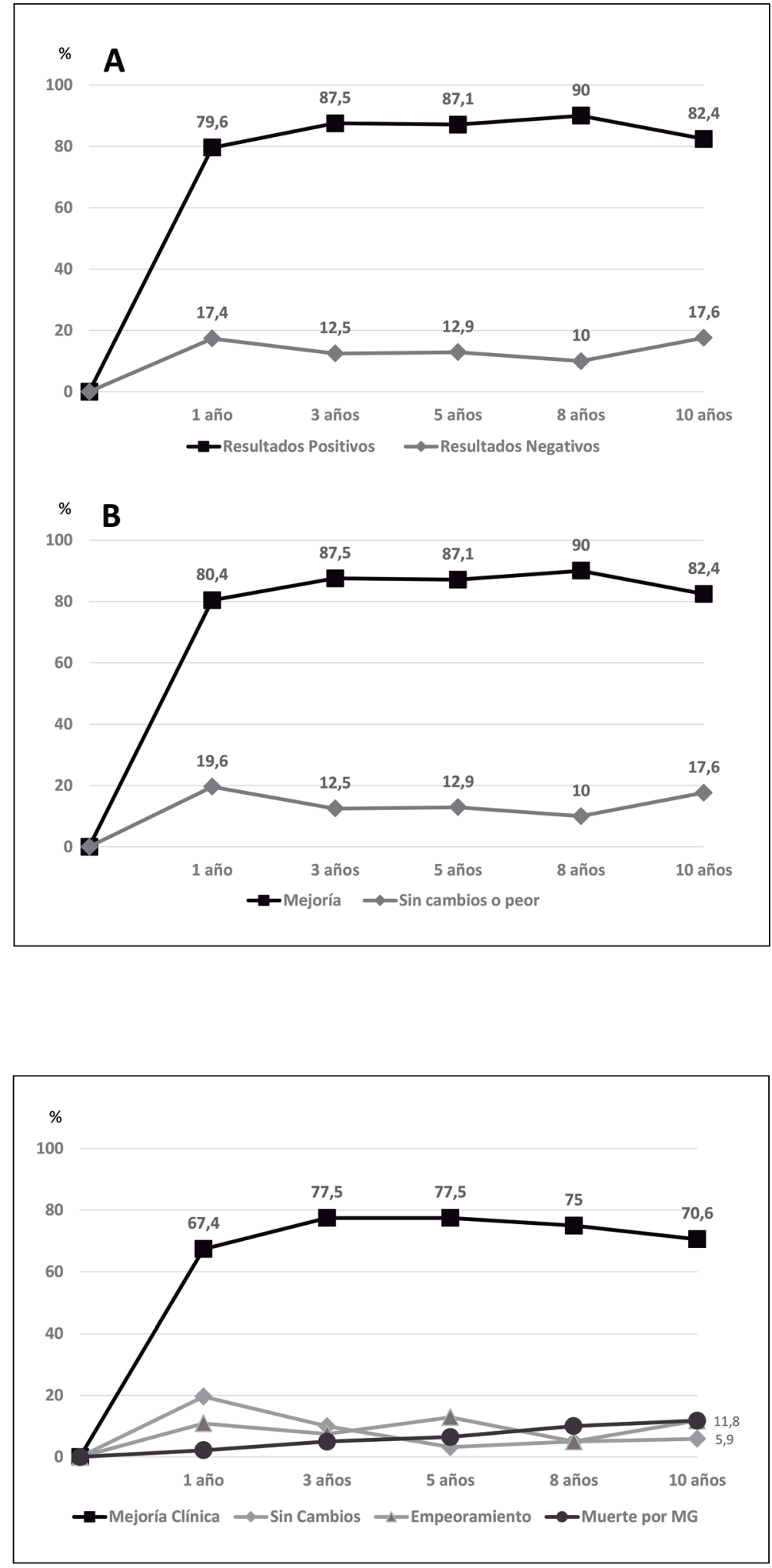

Figura 3. A. Evolución en pacientes timectomizados según clasificación de Zielinski22 a 10 años post timectomía. B. Evolución en pacientes timectomizados según escala de DeFilippi²3 a 10 años post timectomía.

Figura 4. Evolución clínica en pacientes timectomizados según clasificación Myasthenia Gravis Foundation of America Post Intervention Status a 10 años post timectomía. 


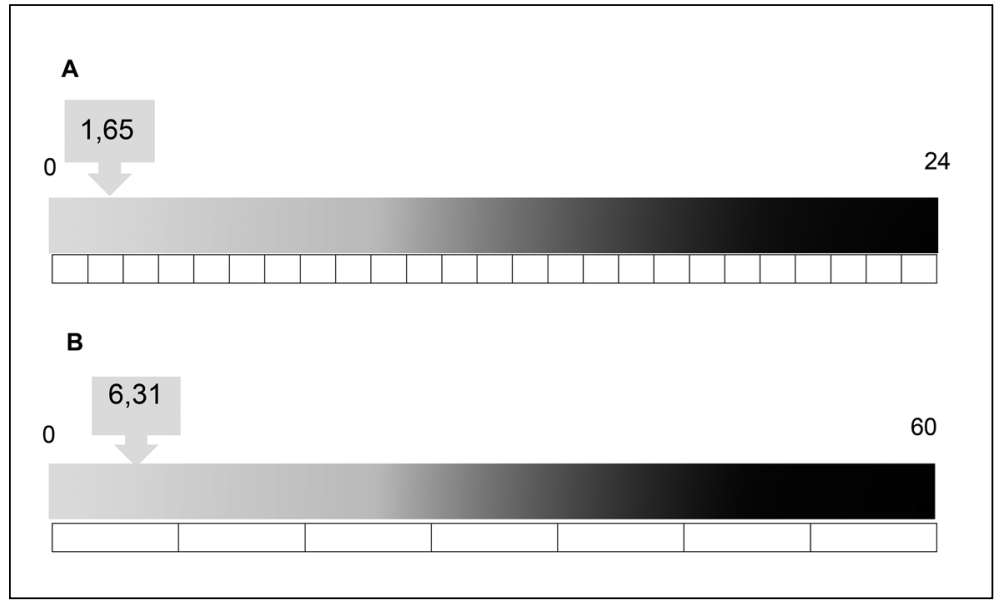

Figura 5. A. Promedio resultado encuesta Myasthenia Gravis Activities of daily living ${ }^{24}$. B. Promedio resultado encuesta Myasthenia Gravis Quality of life scale $15^{25}$.

\section{Discusión}

La timectomía, desde principios del siglo XX, ha sido una de las opciones terapéuticas para el tratamiento de la MG. Influye positivamente en la evolución de esta, al erradicar una fuente de sensibilización y producción de anticuerpos, lo que se correlaciona con el alto porcentaje de anomalías encontradas a la histología (hiperplasia-timoma). Reciente evidencia demuestra claramente que la timectomía y la prednisona fueron superiores al uso de prednisona aislada en pacientes con AChR-MG no timomatosa ${ }^{20,21}$, dicho estudio proporciona evidencia de clase I en favor de la TTEE en MG y tendrá un profundo impacto en la práctica clínica ${ }^{26}$.

$\mathrm{Al}$ observar las características de nuestra serie, estas fueron mayoritariamente mujeres jóvenes, con miastenia generalizada (clasificación IIA-IIB de Osserman) que no superaban los 3 años en promedio de evolución de la MG al momento de la cirugía, similar a lo observado en la literatura internacional ${ }^{20,27-31}$.

Utilizamos la TTEE dada la evidencia existente al día de hoy, en donde publicaciones relevantes y recomendaciones internacionales ${ }^{14,15,20}$ sugieren dicha técnica, ya que permite una resección máxima y reproducible de todo el tejido tímico y adiposo (incluido el tejido tímico extracapsular), minimizando los remanentes de tejido tímico, con una baja morbimortalidad y resultados clínicos a largo plazo superiores a otras técnicas ${ }^{15,20,32}$. Si bien, hoy en día se discuten nuevas formas de abordaje menos invasivas, como la cirugía torácica video-asistida (uni o bilateral), esta no ha logrado demostrar ser superior, en cuanto a resultados alejados a la TTEE en las diferentes revisiones ${ }^{33-35}$. Es necesario nuevos ensayos randomizados para establecer las indicaciones de los enfoques menos invasivos, así como el papel de la timectomía en subgrupos de MG como la de comienzo tardío (sobre los 60 años).

Los resultados inmediatos obtenidos fueron satisfactorios al no registrarse mortalidad inmediata, con una baja morbilidad, semejante a los observado en otras publicaciones donde la mortalidad peri-operatoria es de $0 \%-1 \%$, con tasa de complicaciones que van desde $6 \%$ hasta $33 \%{ }^{20,28-31}$.

El estudio histopatológico de las piezas quirúrgicas demostró principalmente hiperplasia tímica apoyando la teoría del rol inmunológico que posee el timo en la evolución de la MG y el éxito en la mejoría clínica que tiene su extirpación. Se encontró neoplasia epitelial tímica (timoma) en similar proporción a otras series (10\%-20\%).

Al comparar la serie total de pacientes con el subgrupo no timomatoso, se observó que estos últimos presentaban una mejor evolución a largo plazo; confirmando lo expuesto en otras series que lo pacientes con timoma y MG poseen características diferentes dado que su pronóstico neurológico es peor, presentando mayor tasa de persistencia de síntomas, crisis miasténicas y requerimientos 
altos de dosis de anticolinesterásicos. Sin embargo, las neoplasias epiteliales del timo asociada a MG presentan una mayor supervivencia respecto a las otras neoplasias tímicas, esto podría explicarse por la precocidad de los síntomas miasténicos que manifiestan, lo que permite un diagnóstico y tratamiento más oportuno ${ }^{36,37}$.

$\mathrm{Al}$ analizar los resultados a largo plazo observamos una disminución significativa de la necesidad de uso de medicamentos, que se observan a partir del $1^{\text {er }}$ año de seguimiento y se mantiene a lo largo del tiempo. Lo anterior se refleja en las múltiples clasificaciones o escalas utilizadas que permitieron objetivar el progreso de los pacientes, dando resultados satisfactorios en todas ellas, logrando tasas de mejoría y remisión clínica que se mantuvieron a lo largo de los 10 años; dichos resultados fueron comparables a los expuestos en diferentes series que van desde $46 \%-81 \%$ y $17 \%$ $46 \%$ respectivamente ${ }^{16,20-30,32}$. En las encuestas de calidad de vida (MG-ADL y MGQOL-15) se obtuvieron promedios de puntajes bajos, lo que se correlaciona con los satisfactorios resultados obtenidos en las diferentes clasificaciones; cabe destacar que es importante utilizarlos dado su brevedad y facilidad de realizar, junto a la alta sensibilidad que poseen en correlacionar en el tiempo la mejoría o empeoramiento clínico ${ }^{38,39}$.

En la literatura existe un variado número de clasificaciones o encuestas, que intentan objetivar la evolución de los tratamientos asociados a la MG, muchos de ellos tratan de integrar una evaluación neurológica y la percepción de los pacientes en cuanto a sus limitaciones y/o síntomas generados por la MG, sin embargo, la definición de términos tales como mejoría o remisión, no siempre es la misma o simplemente no se explicita, es más, existen grupos que crean sus propias clasificaciones sin muchas veces definir los conceptos utilizados, lo que conlleva a que se hagan comparaciones un tanto subjetivas entre las diferentes series, es por esta razón que utilizamos diferentes clasificaciones cuyos términos están bien definidos, siendo estos utilizados y validados internacionalmente; para así minimizar dicho problema.

Las posibles limitaciones de este artículo son la inclusión de pacientes con MG ocular que, si bien las recomendaciones apuntan hacia utilizar solo tratamiento médico, se ha visto a la timectomía como tratamiento posible y efectivo, en subgrupos de pacientes que presenten resistencia o intolerancia a tratamiento médico, dependencia a altas dosis de corticosteroides y progresión de la enfermedad, siendo este último hecho importante dado que $50 \%$ de las MG ocular progresará a miastenia generalizada dentro de los primeros dos años, por lo que parece lógico plantear la timectomia en ellos. Al revisar nuestros pacientes timectomizados con MG ocular observamos que sus indicaciones quirúrgicas fueron porque en uno de ellos se asociaba a timoma y el resto pertenecía a alguno de los subgrupos antes mencionados.

Un reciente meta análisis muestra resultados favorables de la timectomía en pacientes con MG ocular, sin embargo, la heterogeneidad de los grupos en cuanto a selección de los pacientes y respuesta de estos, requiere que se realicen estudios randomizados y multicéntricos para aclarar que grupo efectivamente se beneficia de esta cirugía ${ }^{40}$.

Una segunda limitación son los criterios de selección para cirugía, ya que estos fueron dados por la evaluación clínica, esto se debe a que en nuestro medio no se cuenta con la posibilidad de la detección de anticuerpos para la selección de pacientes. Sin embargo, considerando que, en una reciente revisión en $\mathrm{MG}$, se confirma una correlación entre el nivel de AChR en el suero y el grado de hiperplasia tímica ${ }^{41}$, por lo que el dato de la alta frecuencia de hiperplasia tímica en los pacientes no timomatosos en nuestra serie $(81,3 \%)$ podría explicar que nuestros resultados sean comparables con series internacionales que utilizaron la medición de anticuerpos como criterio de inclusión. Creemos que es mandatorio realizarlo cuando se dispone de la accesibilidad a este método diagnóstico.

En conclusión, la TTEE, es un tratamiento con una baja morbimortalidad en los pacientes con MG. Los resultados inmediatos y a largo plazo son satisfactorios logrando una permanente mejoría o remisión de los síntomas en la mayoría de los pacientes independiente de la clasificación utilizada.

Material suplementario en versión digital de la Revista Médica de Chile en: https://scielo. conicyt.cl/

Agradecimientos: Agradecimiento al Dr. Gil I. Wolfe, Profesor de Neurología de State University of New York at Buffalo, por haber revisado nuestros resultados preliminares y apoyarnos en esta publicación. 


\section{Referencias}

1. Gilhus N. Myasthenia Gravis. N Engl Med 2016; 375 (26): 2570-81.

2. Carr A, Cardwell C, McCarron P, McConville J. A systematic review of population based epidemiological studies in myasthenia gravis. BMC Neurol 2010; 10: 46.

3. McGrogan A, Sneddon S, de Vries C. The incidence of myasthenia gravis: a systematic literature review. Neuroepidemiology 2010; 34 (3): 171-83.

4. Oosterhuis $\mathrm{H}$. The natural course of myastenia gravis: a long term follow-up study. J Neurol Neurosurg Psychiatry 1989; 52 (10): 1121-7.

5. Querol L, Illa I. Myasthenia gravis and the neuromuscular junction. Curr Opin Neurol 2013; 26(5): 459-65.

6. Huijbers M, Lipka A, Plomp J, Niks E, van der Maarel $\mathrm{S}$, Verschuuren J. Pathogenic immune mechanisms at the neuromuscular synapse: the role of specific antibody-binding epitopes in myasthenia gravis. J Intern Med 2014; 275 (1): 12-26.

7. Blick S, Vicent A, Palace J. Myasthenia gravis: a clinical-immunological update. J Neurol 2016; 263 (4): 826-34.

8. Weigert C. Pathologisch-anastomischer Beitrag zur Erb'schen Krankheit (Myasthenia gravis). Neurol Centralbl 1901; 20: 597-601.

9. Marx A, Pfister F, Schalke B, Saruhan-Direskeneli G, Melms A, Ströbel P. The different roles of the thymus in the pathogenesis of the various myasthenia gravis Subtypes. Autoimmun Rev 2013; 12 (9): 875-84.

10. Silvestri N, Wolfe G. Myasthenia Gravis. Semin Neurol 2012; 32 (3): 215-26.

11. Blalock A, Mason M, Morgan H, Riven S. Myasthenia gravis and tumors of the thymic region: report of a case in which the tumor was removed. Ann Surg 1939 110: 544.

12. Blalock A, Harvey A, Ford F, Lilienthal J. The treatment of myasthenia gravis by removal of the thymus gland. JAMA 1941; 117: 1529-33.

13. Kirschner P. The history of surgery of the thymus gland. Chest Surg Clin North Am 2000; 1 (10): 153-65.

14. Jaretzki A, Barohn R, Ernstoff R, Kaminski H, Keesey J, Penn A, et al. Myasthenia gravis: recommendations for clinical research standards. Neurology 2000; 55 (1): 16-23.

15. Masaoka A, Yamakawa Y, Niwa H, Fukai I, Kondo S, Kobayashi M, et al. Extended thymectomy for myasthenia gravis patients. A 20-year review. Ann Thorac Surg 1996; 62: 853-9.

16. Seguel E, Fuentealba M, Saldías R, Alarcón E, Gyhra A, González R. Resultados de la timectomía transesternal en miastenia gravis. Rev Chil Cir 2003; 55 (5): 440-4.

17. Jans J, González R. Resultados de la cirugía torácica mínimamente invasiva (vídeo-asistida) en el tratamiento de la Miastenia Gravis. Rev Chil Cir 2013; 65 (1): 64-72.

18. Cea G, Benatar M, Verdugo R, Salinas R. Thymectomy for non-thymomatous myasthenia gravis. Cochrane Database Syst Rev 2013; 10: CD008111.

19. Gronseth G, Barohn R. Practice parameter: thymectomy for autoimmune myasthenia gravis (an evidence-based review): report of the Quality Standards Subcommittee of the American Academy of Neurology. Neurology 2000; 55 (1): 7-15.

20. Wolfe G, Kaminski H, Aban I, Minisman G, Kuo H, Marx A, et al. Randomized trial of thymectomy in myasthenia gravis. N Engl J Med 2016; 375: 511-22.

21. Wolfe G, Kaminski H, Aban I, Minisman G, Kuo H, Marx A, et al. Randomized trial of thymectomy in myasthenia gravis. N Engl J Med 2016; 375:511-22. Correction from: N Engl J Med 2017; 376: 2097.

22. Zielinski M, Kuzdzal J, Szlubowski A, Soja, J. Comparison of Late Results of Basic Transsternal and Extended Transsternal Thymectomies in the Treatment of Myasthenia Gravis. Ann Thorac Surg 2004; 78 (1): 253-8.

23. DeFilippi V, Richman D, Ferguson M. Transcervical thymectomy for myasthenia gravis. Ann Thorac Surg. 1994; 57 (1): 194-7.

24. Wolfe G, Herbelin L, Nations S, Foster B, Bryan W, Barohn R. Myasthenia gravis activities of daily living profile. Neurology 1999; 52 (7): 1487-9.

25. Burns T, Conaway M, Cutter G, Sanders D; Muscle Study Group. Less is more, or almost as much: a 15-item quality-of-life instrument for myasthenia gravis. Muscle Nerve 2008; 38 (2): 957-63.

26. Evoli A. Myasthenia gravis: new developments in research and treatment. Curr Opin Neurol 2017; 30 (5): 464-70.

27. Yu S, Li F, Chen B, Lin J, Yang M, Fu X, et al. Eight-year follow-up of patients with myasthenia gravis after thymectomy. Acta Neurol Scand 2015; 131 (2): 94-101.

28. Roth T, Ackermann R, Stein R, Inderbitzi R, Rösler K, Schmid R. Thirteen years follow-up after radical transsternal thymectomy for myasthenia gravis. Do short-term results predict long-term outcome?. Eur J Cardio-Thorac 2002; 21 (4): 664-70.

29. Bachmann K, Burkhardt D, Schreiter I, Kaifi J, Schurr $\mathrm{P}$, Busch C, et al. Thymectomy is more effective than conservative treatment for myasthenia gravis regarding outcome and clinical improvement. Surgery 2009; 145 (4): 392-8.

30. Huang C, Li W, Lee P, Kao K, Chou T, Wu M, et al. Analysis of outcomes following surgical treatment of 
thymolipomatous myasthenia gravis: comparison with thymomatous and non-thymomatous myasthenia gravis. Interact CardioVasc Thorac Surg 2014; 18 (4): 475-81.

31. Luo Y, Pan D, Chen F, Zhu M, Wang J, Zhang M. Effectiveness of Thymectomy in Non-thymomatous Myasthenia Gravis: a Systematic Review. J Huazhong Univ Sci Technol 2014; 34 (6): 942-9.

32. Kattach H, Anastasiadis K, Cleuziou J, Bucley C, Shine B, Pillai R, et al. Transsternal Thymectomy for Myasthenia Gravis: Surgical Outcome. Ann Thorac Surg 2006; 81 (1): 305-8.

33. Qi K, Wang B, Wang B, Zhang LB, Chu X. Video-assisted thoracoscopic surgery thymectomy versus open thymectomy in patients with myasthenia gravis: a meta-analysis. Acta Chir Belg 2016; 116 (5): 282-8.

34. Zahid I, Sharif S, Routledge T, Scarci M. Video-assisted thoracoscopic surgery or transsternal thymectomy in the treatment of myasthenia gravis? Interact CardioVasc Thorac Surg 2011; 12 (1): 40-6.

35. Meyer D, Herbert M, Sobhani N, Tavakolian P, Duncan A, Bruns M, et al. Comparative clinical outcomes of thymectomy for myasthenia gravis performed by extended transsternal and minimally invasive approaches. Ann Thorac Surg 2009; 87 (2): 385-90.
36. Tsinzerling N, Lefvert A, Matell G, Pirskanen-Matell R. Myasthenia gravis: a long-term follow-up study of Swedish patients with specific reference to thymic histology. J Neurol Neurosurg Psychiatry 2007; 78 (10): 1109-12.

37. González R, Santolaya R, Jadue A, Prats R, Mordojovich G, Rodríguez P. Neoplasias epiteliales del timo: timoma y carcinoma tímico. Caracterización, tratamiento y variables asociadas a supervivencia. Rev Chil Cir 2012; 64 (6): 535-45.

38. Muppidi, Wolfe G, Conaway M, Burns T, MG composite and MG-QOL15 study group. MG-ADL: still a relevant outcome measure. Muscle Nerve 2011; 44 (5): 727-31.

39. Muppidi S. The myasthenia gravis-specific activities of daily living profile. Ann N Y Acad Sci 2012; 1274: 114-9.

40. Zhu K, Li J, Huang X, Xu W, Liu W, Chen J, et al. Thymectomy is a beneficial therapy for patients with non-thymomatous ocular myasthenia gravis: a systematic review and meta-analysis. Neurol Sci 2017; 38 (10): 1753-60.

41. Truffault F, de Montpreville V, Eymard B, Sharshar T, Le Panse R, Berrih-Aknin S. Thymic Germinal Centers and Corticosteroids in Myasthenia Gravis: an Immunopathological Study in 1035 Cases and a Critical Review. Clin Rev Allergy Immunol 2017; 52(1): 108-24. 